Infinite Dimensional Analysis, Quantum Probability and Related Topics

Vol. 5, No. 3 (2002) 1-8

(C) World Scientific Publishing Company

\title{
CANONICAL REPRESENTATION OF STATIONARY QUANTUM GAUSSIAN PROCESSES
}

\author{
LUIGI ACCARDI \\ Centro Vito Volterra, Universidat de Roma Tor Vergata, Rome, Italy \\ accardi@volterra.mat.uniroma2.it \\ YUJI HIBINO \\ Faculty of Science and Engineering, Saga University, Saga, Japan
}

Received 23 October 2001

Revised 27 February 2002

\begin{abstract}
Stimulated by the quantum generalization of the canonical representation theory for Gaussian processes in Ref. 1, we first give the representations (not necessarily canonical) of two stationary Gaussian processes $X$ and $Y$ by means of white noises $q_{t}$ and $p_{t}$ with no assumptions on their commutator. We then assume that $q_{t}+i p_{t}$ annihilates the vacuum state and prove that the representations are the joint Boson-Fock ones if and only if $X$ and $Y$ have a scalar commutator.
\end{abstract}

Keywords:

\section{Introduction}

The structure theory of stationary stochastic processes and its closed connections with complex analysis (cf. Refs. 2, 4 and Sec. 1 below) are now well understood. The program to extend this to that of the quantum case has been proposed in Ref. 1.

Recall that any classical stochastic process $X_{t}$ can be realized as an operator on a Hilbert space $\mathcal{H}$ with a unit vector $\Phi$ through the relation

$$
\left\langle\Phi, f\left(X_{t}\right) \Phi\right\rangle=E\left[f\left(X_{t}\right)\right]
$$

for any Borel function $f$ of the process $X_{t}$ such that $\Phi$ is in the domain of $f\left(X_{t}\right)$.

In the general case, we will identify a stochastic process with a family of symmetric operators $X_{t}$ defined on a common dense subspace of a Hilbert space $\mathcal{H}$. A process is called classical if the operators $X_{t}$ commute in the sense that

$$
\left\langle X_{s} \xi, X_{t} \eta\right\rangle=\left\langle X_{t} \xi, X_{s} \eta\right\rangle
$$

for each $\xi, \eta$ in the common domain and for each real $s, t$. If $\Phi$ is a vector in this domain, the $\Phi$-marginal distribution of a classical process $X_{t}$ are defined by (0). In this sense one can speak of $\Phi$-Brownian motion. 
In Sec. 1, we review some properties on the canonical representation of classical stationary processes. From the viewpoint of the canonical representation theory, the most remarkable facts are that a stationary process always has the canonical representation and that the canonical representation kernel can be constructed by its spectral density function.

In Sec. 2, we recall some results obtained in Ref. 1 . We give a necessary condition on the commutator of two given stationary processes, for the existence of a quantum canonical representation in the sense of Ref. 1 .

Section 3 is the main part of this paper. We find that a standard $\Phi$-white noise with a scalar commutator is nothing but a Boson-Fock white noise. Consequently, if two given stationary processes have a scalar commutator, their representations by standard white noises are already a joint Boson-Fock representation. This result means that the necessary condition obtained in Sec. 2 is also sufficient.

\section{Classical Case}

It is known that every purely nondeterministic mean-continuous stationary centered Gaussian process $Y_{t}$ can be expressed as

$$
Y_{t}=\int F(t-u) d B_{u}=\int F(t-u) w_{u} d u=(F * w)_{t},
$$

where $w_{u}$ is a standard white noise and $F$ is a square-integrable function with support in $[0, \infty)$. This representation is called a moving average representation.

From the viewpoint of the canonical representation theory, ${ }^{4}$ the most remarkable point for a stationary process is that it always has a canonical representation. That is to say, among the representations of the form (1), there exists an essentially unique representation satisfying $\mathcal{B}_{t}(Y)=\mathcal{B}_{t}(B)\left(=\mathcal{B}_{t}(w)\right)$ for each $t \in \mathbb{R}$, where $\mathcal{B}_{t}(Y)$ is the $\sigma$-field generated by $Y_{s}, s \leq t$.

In this case, it is known that the spectral measure is absolutely continuous and that its spectral density function $h(\lambda)$ satisfies

$$
\int_{-\infty}^{\infty} \frac{\log h(\lambda)}{1+\lambda^{2}} d \lambda>-\infty
$$

In the above, the spectral measure stands for the measure whose Fourier transform is given by the time correlation function of the process. The image of the space $L^{2}[0, \infty)$ under the inverse Fourier transform, being identified with the space of the square integrable functions supported by $[0,+\infty)$, is identified with the Hardy class $\mathbf{H}_{2}$ in the lower half-plane (Ref. 2). Any element $c(\lambda)$ of $\mathbf{H}_{2}$ has a unique decomposition of the form

$$
c(\lambda)=C c_{O}(\lambda) c_{I}(\lambda)
$$


with

$$
\begin{aligned}
C & =e^{i \gamma}, \\
c_{O}(\lambda) & =\sqrt{2 \pi} \exp \left\{-\frac{1}{\pi i} \int_{-\infty}^{\infty} \frac{1+w \lambda}{w-\lambda} \frac{\log h(w)}{1+w^{2}} d w\right\}, \\
c_{I}(\lambda) & =\Pi(\lambda) \exp \left\{\frac{1}{\pi i} \int_{-\infty}^{\infty} \frac{1+w \lambda}{w-\lambda} d \beta(w)-i \alpha \lambda\right\},
\end{aligned}
$$

where $\gamma$ is a real constant, $\Pi$ is a Blaschke product, $\alpha$ is a non-negative constant and $\beta$ is a nondecreasing function of bounded variation with derivative vanishing almost everywhere. The components $c_{O}(\lambda)$ and $c_{I}(\lambda)$ are called the outer function and the inner function of $c(\lambda)$, respectively. It is known that $\left|c_{O}(\lambda)\right|^{2}=2 \pi h(\lambda)$ and $\left|c_{I}(\lambda)\right|=1$ for $\lambda \in \mathbb{R}$.

The necessary and sufficient condition for (1) to be a canonical representation is $c(\lambda)=C c_{O}(\lambda)$ (Ref. 5). Therefore the noncanonical property of the representation (1) comes from the inner function, hence the difference between the inverse Fourier transform of the kernel of the canonical representation and the kernel of a noncanonical representation is just the multiplication by a function of unit modulus.

\section{Necessary Condition}

A Boson-Brownian motion is defined by a pair of classical $\Phi$-Brownian motions $Q_{s}$ and $P_{t}$ satisfying (on a common dense domain):

$$
\left[Q_{s}, P_{t}\right]=i(s \wedge t) .
$$

We can introduce the representations of white noises as

$$
Q_{s}=\int_{0}^{s} q_{v} d v, \quad P_{t}=\int_{0}^{t} p_{u} d u
$$

where $q_{s}$ and $p_{t}$ satisfy

$$
\left[q_{s}, p_{t}\right]=i \delta(s-t) .
$$

If, in addition, $q_{s}, p_{t}$ satisfy condition (13) below, then one speaks of the Boson-Fock white noise.

Definition 1. ${ }^{1}$ Two classical stationary Gaussian processes $X, Y$ admit a joint Boson-Fock representation, if $X$ and $Y$ can be represented by using a Boson-Fock white noise $\left(q_{s}, p_{t}\right)$ as follows:

$$
X_{s}=\int G(s-v) q_{v} d v=(G * q)_{s}
$$

and

$$
Y_{t}=\int F(t-u) p_{u} d u=(F * p)_{t},
$$


respectively. This representation is called canonical if both (7) and (8) are isomorphic to the canonical representation of $X$ and $Y$, respectively.

Theorem 1. ${ }^{1}$ If $X$ and $Y$ have a joint Boson-Fock representation in the form (7) and (8), then the commutator of $X$ and $Y$ is expressed as

$$
\left[X_{s}, Y_{t}\right]=i \Theta(s-t)
$$

where $\Theta=(\hat{G} \check{F})^{\vee}$. In particular $\Theta$ is a scalar function.

Proof. It is clear that

$$
\begin{aligned}
{\left[X_{s}, Y_{t}\right] } & =\iint G(s-v) F(t-u)\left[q_{v}, p_{u}\right] d u d v \\
& =i \iint G(s-v) F(t-u) \delta(v-u) d u d v \\
& =i \int G(s-u) F(t-u) d u \\
& =i \int G(s-u) F_{-}(u-t) d u \quad\left(\text { where } F_{-}(x):=F(-x)\right) \\
& =i\left(G * F_{-}\right)(s-t) \\
& =i\left(\hat{G} \hat{F}_{-}\right)^{\vee}(s-t) \\
& =i(\hat{G} \check{F})^{\vee}(s-t) .
\end{aligned}
$$

Theorem 1 suggests to introduce the class of functions:

$$
\mathcal{D}=\left\{(\hat{G} \check{F})^{\vee} ; G, F \in L^{2}[0, \infty)\right\} .
$$

Then that $\left[X_{s}, Y_{t}\right]$ belongs to $i \mathcal{D}$ is a necessary condition for two given classical processes to have a joint Boson-Fock representation. Besides, for

$$
\mathcal{D}_{0}=\left\{(\hat{G} \check{F})^{\vee} ; \text { both } G \text { and } F \text { are canonical kernels }\right\}(\subsetneq \mathcal{D}),
$$

that $\left[X_{s}, Y_{t}\right]$ belongs to $i \mathcal{D}_{0}$ is a necessary condition for two given classical processes to have a canonical joint Boson-Fock representation.

In this paper, we assume that $X$ and $Y$ have a scalar commutator and we look for conditions under which they admit a joint Boson-Fock representation.

\section{Scalar Commutators}

We put $a_{t}=q_{t}+i p_{t}$ and $a^{\dagger}{ }_{t}=q_{t}-i p_{t}$, and suppose that

$$
a_{t} \Phi=0
$$

and that the vectors $a_{t}^{+n} \Phi(n \in \mathbb{N})$ are total in the sense of distributions. 
Lemma 2. If $\left[q_{s}, p_{t}\right]=i g(s-t)$ for a scalar distribution $g$ and (13), then $g$ is a real even function.

Proof. Since

$$
-i \bar{g}(s-t)=\left[q_{s}, p_{t}\right]^{*}=\left[p_{t}, q_{s}\right]=-\left[q_{s}, p_{t}\right]=-i g(s-t),
$$

we know that $g$ must be real. Moreover,

$$
\left[a_{s}, a_{t}^{\dagger}\right]=\left[q_{s}+i p_{s}, q_{t}-i p_{t}\right]=g(s-t)+g(t-s)
$$

and, denoting $a(\varphi)=\int \varphi(s) a_{s} d s$ for any test function $\varphi$, we deduce from (13) and (15) that

$$
\begin{aligned}
a(\varphi)\left(a^{\dagger}(\psi)\right)^{n} \Phi & =\sum_{k=0}^{n-1}\left(a^{\dagger}(\psi)\right)^{k}\left[a(\varphi), a^{\dagger}(\psi)\right]\left(a^{\dagger}(\psi)\right)^{n-k-1} \Phi \\
& =n\left[a(\varphi), a^{\dagger}(\psi)\right]\left(a^{\dagger}(\psi)\right)^{n-1} \Phi .
\end{aligned}
$$

By using this formula, for any test functions $\varphi, \psi$ and $\chi$,

$$
\begin{aligned}
& a(\chi) a(\varphi)\left(a^{\dagger}(\psi)\right)^{n} \Phi=n(n-1)\left[a(\varphi), a^{\dagger}(\psi)\right]\left[a(\chi), a^{\dagger}(\psi)\right]\left(a^{\dagger}(\psi)\right)^{n-2} \Phi, \\
& a(\varphi) a(\chi)\left(a^{\dagger}(\psi)\right)^{n} \Phi=n(n-1)\left[a(\chi), a^{\dagger}(\psi)\right]\left[a(\varphi), a^{\dagger}(\psi)\right]\left(a^{\dagger}(\psi)\right)^{n-2} \Phi .
\end{aligned}
$$

Since $\left[a(\varphi), a^{\dagger}(\psi)\right]$ is scalar, we obtain that $a(\chi) a(\varphi)=a(\varphi) a(\chi)$. Therefore, on the total set of vectors $a_{t}^{+n} \Phi$, one has

$$
\left[a_{s}, a_{t}\right]=\left[q_{s}+i p_{s}, q_{t}+i p_{t}\right]=-g(s-t)+g(t-s)=0 .
$$

This means that $g$ is even.

Proposition 3. Suppose that $q_{s}$ and $p_{t}$ are standard white noises satisfying condition (13), and that $\left[q_{s}, p_{t}\right]=i g(s-t)$ for a scalar distribution $g$. Then $g=\delta$.

Proof. Because of Lemma 2,

$$
\left[a_{s}, a_{t}^{\dagger}\right]=\left[q_{s}+i p_{s}, q_{t}-i p_{t}\right]=2 g(s-t)
$$

or equivalently

$$
\left[a(\varphi), a^{\dagger}(\varphi)\right]=2 \iint \varphi(s) g(s-t) \varphi(t) d s d t
$$

From (16),

$$
a(\varphi)\left(a^{\dagger}(\varphi)\right)^{n} \Phi=n\left[a(\varphi), a^{\dagger}(\varphi)\right]\left(a^{\dagger}(\varphi)\right)^{n-1} \Phi,
$$


therefore $\left\langle\left(a^{\dagger}(\varphi)\right)^{n} \Phi,\left(a^{\dagger}(\varphi)\right)^{n} \Phi\right\rangle=n !\left[a(\varphi), a^{\dagger}(\varphi)\right]^{n}$. Thus

$$
\begin{aligned}
\left\langle e^{a^{\dagger}(\varphi)} \Phi, e^{-a^{\dagger}(\varphi)} \Phi\right\rangle & =\sum_{m, n} \frac{1}{m !} \frac{(-1)^{n}}{n !}\left\langle\left(a^{\dagger}(\varphi)\right)^{m} \Phi,\left(a^{\dagger}(\varphi)\right)^{n} \Phi\right\rangle \\
& =\sum_{n} \frac{(-1)^{n}}{(n !)^{2}}\left\langle\left(a^{\dagger}(\varphi)\right)^{n} \Phi,\left(a^{\dagger}(\varphi)\right)^{n} \Phi\right\rangle \\
& =\sum_{n} \frac{(-1)^{n}}{n !}\left[a(\varphi), a^{\dagger}(\varphi)\right]^{n} \\
& =e^{-\left[a(\varphi), a^{\dagger}(\varphi)\right]} .
\end{aligned}
$$

Therefore $e^{ \pm a^{\dagger}(\varphi)} \Phi=\sum_{n} \frac{( \pm 1)^{n}}{n !}\left(a^{\dagger}(\varphi)\right)^{n} \Phi$ is well-defined, in the sense that the series on the right converges in norm in $\mathcal{H}$ and from the known identity

$$
e^{a(\varphi)} e^{-a^{\dagger}(\varphi)}=e^{a(\varphi)-a^{\dagger}(\varphi)-\frac{1}{2}\left[a(\varphi), a^{\dagger}(\varphi)\right]}=e^{-\frac{1}{2}\left[a(\varphi), a^{\dagger}(\varphi)\right]} e^{a(\varphi)-a^{\dagger}(\varphi)},
$$

it follows that

$$
e^{a(\varphi)-a^{\dagger}(\varphi)}=e^{\frac{1}{2}\left[a(\varphi), a^{\dagger}(\varphi)\right]} e^{a(\varphi)} e^{-a^{\dagger}(\varphi)} .
$$

Therefore, taking the $\Phi$-expectation value on both sides, we obtain

$$
\left\langle\Phi, e^{a(\varphi)-a^{\dagger}(\varphi)} \Phi\right\rangle=e^{\frac{1}{2}\left[a(\varphi), a^{\dagger}(\varphi)\right]}\left\langle e^{a^{\dagger}(\varphi)} \Phi, e^{-a^{\dagger}(\varphi)} \Phi\right\rangle .
$$

This and (23) imply that

$$
\left\langle\Phi, e^{a(\varphi)-a^{\dagger}(\varphi)} \Phi\right\rangle=e^{\frac{1}{2}\left[a(\varphi), a^{\dagger}(\varphi)\right]} e^{-\left[a(\varphi), a^{\dagger}(\varphi)\right]}=e^{-\frac{1}{2}\left[a(\varphi), a^{\dagger}(\varphi)\right]} .
$$

Since, by assumption, $p_{t}=\frac{1}{2 i}\left(a_{t}-a^{\dagger} t\right)$ is a standard $\Phi$-white noise, $a(\varphi)-a^{\dagger}(\varphi)$ is a Gaussian random variable with mean 0 and variance $2 \int \varphi(s)^{2} d s$. Thus we know that

$$
\left\langle\Phi, e^{a(\varphi)-a^{\dagger}(\varphi)} \Phi\right\rangle=e^{-\int \varphi(s)^{2} d s} .
$$

So, $\int \varphi(s)^{2} d s=\frac{1}{2}\left[a(\varphi), a^{\dagger}(\varphi)\right]=\iint \varphi(s) g(s-t) \varphi(t) d s d t$ holds for any test function $\varphi$. Therefore

$$
g(s-t)=\delta(s-t)
$$

Theorem 4. Let $X$ and $Y$ be classical stationary Gaussian processes represented in the forms (7) and (8) by using standard white noises $q$ and $p$ satisfying condition (13). If their commutator is $\left[X_{s}, Y_{t}\right]=i \Theta(s-t)$, for some scalar function $\Theta$, then (7) and (8) are a joint Boson-Fock representation of $X$ and $Y$.

Proof. Since $\hat{X}=(G * q)^{\wedge}=\hat{G} \hat{q}$,

$$
q=\left(\frac{1}{\hat{G}} \hat{X}\right)^{\vee}=\left(\frac{1}{\hat{G}}\right)^{\vee} * X
$$


Similarly, $\check{Y}=(F * p)^{\vee}=\check{F} \check{p}$,

$$
p=\left(\frac{1}{\check{F}} \check{Y}\right)^{\wedge}=\left(\frac{1}{\check{F}}\right)^{\wedge} * Y
$$

Because $\hat{G}$ and $\check{F}$ are analytic in the upper and lower half-plane, respectively, they do not vanish except for a set of measure zero. Therefore

$$
\begin{aligned}
{\left[q_{s}, p_{t}\right] } & =\iint\left(\frac{1}{\hat{G}}\right)^{\vee}(s-v)\left(\frac{1}{\check{F}}\right)^{\wedge}(t-u)\left[X_{v}, Y_{u}\right] d u d v \\
& =i \iint\left(\frac{1}{\hat{G}}\right)^{\vee}(s-v)\left(\frac{1}{\check{F}}\right)^{\wedge}(t-u) \Theta(v-u) d u d v \\
& =i \int\left(\frac{1}{\check{F}}\right)^{\wedge}(t-u)\left(\left(\frac{1}{\hat{G}}\right)^{\vee} * \hat{\Theta}^{\vee}\right)(s-u) d u \\
& =i \int\left(\frac{1}{\check{F}}\right)^{\vee}(u-t)\left(\frac{\hat{\Theta}}{\hat{G}}\right)^{\vee}(s-u) d u \\
& =i\left(\left(\frac{1}{\check{F}}\right)^{\vee} *\left(\frac{\hat{\Theta}}{\hat{G}}\right)^{\vee}\right)(s-t) \\
& =i\left(\frac{\hat{\Theta}}{\hat{G} \check{F}}\right)^{\vee}(s-t),
\end{aligned}
$$

which is a scalar and therefore, thanks to Proposition 3, we can conclude that

$$
\left(\frac{\hat{\Theta}}{\hat{G} \check{F}}\right)^{V}=\delta(s-t) .
$$

Thus (7) and (8) give a joint Boson-Fock representation of $X$ and $Y$.

Remark 1. This result tells that $\hat{\Theta}=\hat{G} \check{F}$. If $X$ and $Y$ have a scalar commutator, it should be in $i \mathcal{D}$. So we can say that the necessary and sufficient condition for $X$ and $Y$ to admit a joint Boson-Fock representation is that $\left[X_{s}, Y_{t}\right]$ belongs to $i \mathcal{D}$.

\section{Concluding Remark}

The following problem was considered in Ref. 1:

For two given processes $X$ and $Y$ which admit a joint Boson-Fock representation with a scalar commutator, under which condition is it possible to construct the canonical representation of $X$ and $Y$ ? In Ref. 1 it was proved that, if: (i) both $X$ and $Y$ are multiple Markov in the sense of $\mathrm{Hida}^{3}$; (ii) their spectral functions have no zeros on the real axis; then such a reconstruction is possible.

As we have seen in Sec. 2, the condition that the commutator of $X$ and $Y$ belongs to $i \mathcal{D}_{0}$ is necessary. However, Theorem 4 tells that we can always construct 
a joint Boson-Fock representation if a scalar commutator $\left[X_{s}, Y_{t}\right]$ belongs to $i \mathcal{D}$. If the commutator $\left[X_{s}, Y_{t}\right]$ belongs to $i \mathcal{D}_{0}$ and the representations of $X$ and $Y$ are noncanonical, then the inner function of $\hat{G}$ must be canceled by the inner function of $\check{F}$, namely $X$ and $Y$ should have the same noncanonical part. It follows that, if the commutator $\left[X_{s}, Y_{t}\right]$ belongs to $i \mathcal{D}_{0}$ and if one of two representations (7) and (8) is canonical, then the other one must be canonical. So it is sufficient to check the canonical property for only one of the representations.

\section{Acknowledgment}

This work was supported by Vito Volterra Fellowship 2000.

\section{References}

1. L. Accardi, T. Hida and Win Win Htay, Boson-Fock representations of stochastic processes, Math. Notes 67 (2000) 3-14.

2. H. Dym and H. P. McKean, Gaussian Processes, Function Theory, and the Inverse Spectral Problem (Academic Press, 1976).

3. T. Hida, Canonical representations of Gaussian processes and their applications, Mem. Coll. Sci. Univ. Kyoto Ser. A 33 (1960) 109-155.

4. T. Hida and M. Hitsuda, Gaussian Processes, Representation and Applications (Amer. Math. Soc., 1993).

5. K. Karhunen, Über die struktur stationärer zufälliger funktionen, Ark. f. Mat. 1 (1950) $141-160$. 